\title{
UNA PROPUESTA DE ANÁLISIS LINGÜÍSTICO-POÉTICO DE CUATRO DE LOS CANTARES DE DZITBALCHÉ
}

\author{
Luckro Meléndez GumorrRama \\ IIA, UNAM
}

\section{Introducción}

Los Cantares de Dzitbalché son un grupo de 15 textos y una portada que proceden del pueblo de Dzitbalché, Campeche $e^{2}$ y que fueron escritos durante la época colonial en idioma maya yucateco - lengua perteneciente a la rama yucatecana de la familia lingüistica maya-. Su contenido temático ha sido catalogado como ritual y como el único documento de su época en el género de poesia lírica (Edmoson y Bricker, 1985: 52).

La primera edición de los textos estuvo a cargo de Alfredo Barrera Vásquez (1980), quien también se encargó de la traducción del manuscrito. El valioso trabajo de Barrera consistió en la paleografia, traducción y publicación de éstos. Sin embargo, los Cantares fueron publicados en un orden arbitrario en el que no se realizó un análisis consistente sobre su contenido ritual. Desde una perspectiva literaria, Munro Edmonson (1982) publicó los Cantares con un orden distinto y propuso una traducción alternativa a la de Barrera (1980). Por su parte, Martha llia Nảjera (2007) planteó una nueva articulación de los Cantares con base en su contenido ritual, abordado desde la perspectiva de la religión maya.

La motivación por trabajar estos documentos desde un enfoque linguísticopoético surgió de mi interés por realizar una investigación consistente sobre la poética del maya yucateco colonial. Como es bien sabido, los datos contenidos en las fuentes coloniales pueden servir a los lingüistas para obtener datos escritos de otros momentos de la lengua. Bajo esta perspectiva, podemos estudiar los registros coloniales de la lengua maya desde cualquiera de los niveles de análisis linguistico. En este caso, utilicé los Cantares como corpus lingüistico y me enfoqué en la descripción de la estructura formal y semántica de las formas discursivamente marcadas que fueron expresadas en éstos. A su vez, estas formas discursivas pueden ser contrastadas con distintos textos mayas producidos

\footnotetext{
'Agradezco a Leopoldo Valitas por haber aceptado leer este trabajo y por sus valiosas comen tarios. También agradexco a la linguista Etna Pascacio por toda su ayuda reflejada en la parte de fonetización de los documentos, y a la doctora Carmen Valverde y a la maestra Cristina Buenrostro por sus valiosos comentarios.

${ }^{2}$ Alfredo Barrera Visquez los encontró en la ciudad de Mérida en 1942.
} 
durante el periodo colonial con el fin de encontrar las diferencias discursivas entre los Cantores y los demás textos.

En términos generales, en este estudio me enfoqué en la descripción y anälisis de las figuras literarias llamadas paralelismos, aliteraciones y anáforas en los cantares $4,7,14$ y $15 .{ }^{3} \mathrm{El}$ contenido temático de los textos es de carácter religioso-ritual, pero en éste no me concentraré en el contenido sino en su forma. Sin embargo, sobre los rituales quiero mencionar que si bien es cierto que tenemos algunas menciones en crónicas coloniales sobre éstos acompañados de danza y música, actualmente sólo podemos plantear hipótessis sobre su ejecución. Toda esa información se perdió con el paso del tiempo, por lo que sólo es posible trabajar con la parte escrita de los mismos, de tal manera que aquello relacionado con el performance de los Cantares quedó fuera de esta investigación.

De manera global, el trabajo se ocupa de la descripción y análisis de las figuras literarias llamadas paralelismos, aliteraciones y anáfora de los cantares seleccionados.

\section{Presentación y metodologia de la transcripción del corpus}

En el Apéndice 1, el lector podrá encontrar los cuatro cantares que elegi para este articulo.

En la actualidad, los estudios linguiisticos que se realizan sobre textos coloniales en lenguas indigenas requieren forzosamente de un análisis y sistematización de las grafias representadas en el documento que se esté trabajando. En tanto que dichos documentos carecen de un registro linguiistico sistemático, es necesario establecer los fonemas de la lengua en cuestión con el fin de que los textos conformen un corpus lingüistico analizable desde cualquier nivel de la lengua.

Las grafias representadas en los Cantares de Dzitbalché no muestran una correspondencia con los fonemas de la lengua y. por esta razón, realicé una fonetización del corpus con base en un análisis de linguística histórica buscando dar una interpretación sistemática a los registros. El primer paso para la fonetización de los textos es el establecimiento de la estructura silábica. Los datos linguiisticos contenidos en los Cantares, asi como datos del yucateco actual apuntan a que el yucateco del siglo xvu tiene silabas del tipo CV y CVC y, de acuerdo con la tipologia de Clements y Keiser (1983), es una lengua del tipo III. En el corpus no existe la representación del fonema consonántico glotal / / en ninguna posición. He decidido restituir este fonema en posición onset inicio de palabra y en coda final de palabra tomando como punto de partida datos de la lengua maya actual como en el siguiente ejemplo:

\footnotetext{
${ }^{1}$ Según el orden de presentación de Barrera (1980).
} 
(1) NILCTÉ-HA

nikte? ha?

flor agua

'nenúfar' (Cantar 7 , linea 37)

En el ejemplo ( 1 ) restitui la consonante $/ /$ en coda final de palabra tanto la palabra /nikte?/ 'flor' como en /ha?/ 'agua'. Para realizar la restitución de la consonante glotal / $/$ en coda final de palabra me basé en evidencia estructural de la lengua y también en evidencia léxica. Si bien es cierto que la lengua tiene silabas del tipo $\mathrm{CV}$, algunas palabras no tienen representada la consonante glotal final porque es común que se debilite en estos contextos y que no se registre, ${ }^{4}$ por ello resulta necesario restituir el fonema $/ /$ en ese contexto.

En tanto que esta lengua es del tipo III, es decir, sólo permite silabas del tipo CV y CVC vemos que las silabas desnudas no están permitidas y es necesario restituir el fonema glotal $/ /$ inicial para respetar las estructuras $\mathrm{CV}$ y CVC como en la línea 46 del cantar 4 en el ejemplo (2):

(2) OLIL-VAY-OITIL

7 olil way

"aquif

En cuanto al numero de fonemas en cada posición silábica, encontramos que en onset y coda sólo se permite una consonante como máximo. Los núcleos silábicos pueden ser monomoraicos o bimoraicos, aunque la representación en el documento respecto a los nuicleos bimoraicos en ocasiones no es sistemática. En estos casos, recurri una vez más a la evidencia léxica en el Diccionario Maya Cordemex.

En el maya yucateco no se permiten grupos consonánticos en posición onset inicio de palabra, por lo que cuando tenemos un grupo consonántico representado con las grafias $\langle C$ - K $\rangle$ (Cantar 4 , linea 2 ) reconstruyo el fonema $/ \mathrm{k}^{*} /$ con base en evidencia léxica. Cuando aparecen secuencias con la palatal fricativa $/ 5 /$-consonante, restituyo la secuencia consonante glotal-vocal alta anterior $/ \mathrm{i} /$ precediendo a la palatal fricativa / $/$ /. Si tenemos las grafias $\langle\mathrm{TT}\rangle$, reconstruyo la consonante alveolar oclusiva glotalizada $/ t$ \% El resto de las consonantes glotalizadas fueron indicadas en el registro original.

En cuanto a la consonante aspirada $/ \mathrm{h} /$ no he encontrado suficiente evidencia para saber si realmente se trata de una aspiración o si es una variación en el registro del mismo fonema velar continuo /j/. En el Diccionario Maya Cordemex, no se establece ninguna diferenciación entre los fonemas. En el maya yucateco actual la distinción entre las consonantes $/ \mathrm{h} / \mathrm{y} / \mathrm{j} / \mathrm{no}$ se mantiene, pero no

\footnotetext{
${ }^{4}$ Además de que se trata la consonante glotal no existe en el espanol y para el siglo xue no existia una convención para representar este fonema.
} 
tengo suficientes argumentos para proponer que la distinción no existia en el siglo $\mathrm{xvw}$.

Las grafias $<\mathrm{u}, \mathrm{i}>$ aparecen en posiciones consonánticas y vocálicas. Para representar los fonemas continuos $/ y / y / w /$ en los Cantares se utilizaron las grafias $\langle\mathrm{l}\rangle \mathrm{y}\langle\mathrm{V}\rangle$, respectivamente. Cuando ocupan posición onset o coda, funcionan como consonantes y no como vocales. Considero estas mismas grafias como vocales si su posición es de núcleo de silaba.

En todo el corpus aparecen secuencias de vocales idénticas. Estas secuencias están registradas para todas las calidades vocálicas, es decir, aa, ee, ii, oo, uu, y las interpreto como núcleos bimoraicos. Considerando que no están permitidas las silabas del tipo V ni VC, no se puede tratar de secuencias de núcleos de silabas distintas. Tampoco se pueden considerar como realización fonética de silabas acentuadas, ya que en algunos registros el acento se marca en núcleos monomoraicos.

En el Diccionario Maya Cordemex y otros diccionarios del maya yucateco actual aparecen representadas vocales glotalizadas rearticuladas; sin embargo, en estos textos no existe evidencia en la representación de vocales con la explosión glotal como particularidad prosódica.

\section{La elección de los cuatro cantares, metodología y objetivos}

Debido a que el contenido simbólico, literario y ritual de los Cantares es muy diverso, en este estudio me enfocaré sólo en los cantares $4,7,14$ y 15 . En primer lugar porque exhiben una estructura poètica en la que el uso constante de paralelismos y aliteraciones se vuelve un común denominador y, en segundo lugar, porque comparten una temática especifica, es decir, lo que Näjera $\{2007\rangle$ llamó "rituales de iniciación femenina" y que según la autora, están relacionados y escritos con un mismo fin: la celebración de algún rito de paso.

Sobre el contenido literario de los Contores Edmonson (1982: 175-176) señaló que: "[...] a diferencia de muchos de los fragmentos sobrevivientes de la literatura maya, éstos están, de manera inusual, llenos de expresiones de sentimiento y emoción. Tratan del amor, del abandono, de la belleza de la naturaleza, sexo, temor, y por supuesto, tiempo y muerte".5 Especificamente, sobre los cantares 15, 14, 4 y 7 menciona que son canciones de amor, llenas de erotismo, y que representan un ritual orgiástico (ibid.: 174). Aunque el analisis de Edmonson pretende ser literario, su enfoque está centrado en el contenido temático de la obra y no sobre la forma especifica que ésta toma.

${ }^{5}$ La traducción es mia, en el original: "|...] unlike many of the survining fragments of Mayan literature, they are unusually full of expressions of sentiment and emotion. They deal with love, abandonmerst, the beauty of nature, sex, fear, and of course, time and death". 
La interpretación de estos ritos de paso como canciones de amor es el resultado de un análisis que se realizó bajo los conceptos occidentales de lo que es "literario" y de lo que no pertenece a esta esfera. Éste es un grave error metodológico, ya que muchos estudiosos de documentos coloniales escritos en lenguas indigenas han considerado "literario" cualquiera de estos escritos por el sólo hecho de ser indigena. La consideración de los textos rituales como literarios desde una perspectiva occidental no contempla que éstos no fueron producidos por un individuo - el escritor-, sino que es un producto colectivo, esto es, que fue elaborado por una colectividad en el marco de una tradición religiosa determinada y que la intencionalidad en su elaboración no fue "literaria" sino más bien ritual. El hecho de que se mantengan ciertas formas repetitivas obedece justamente al carácter ritual de los textos y a un ritmo que éstos debieron mantener durante su ejecución. Por estos motivos, las ideas de Edmonson (1982) sobre estos rituales como canciones de amor o como un "ritual orgiástico" no pueden ser aceptadas en tanto que el autor no consideró variables de tipo cultural para realizar su análisis y, en cambio, se dejó guiar por los temas que se tocan en los Cantares desde su perspectiva de lo que es lo literario.

La literatura se ocupa de la obra individual y la lingüistica de la obra colectiva, de la tradición oral, es decir, de lo que Jakobson (1984 |1956) define como folklore, en oposición a la obra individual que produce literatura. Bajo la optica de Jakobson, los Cantores están dentro del ámbito del folklore y no dentro de la literatura y por ello forman parte del campo de estudio de la linguistica y no de la critica literaria. Partiendo de este supuesto, en este trabajo analizaré, desde una perspectiva lingüistica, las formas marcadas del discurso que están enfocadas en la función poética del lenguaje (véase Jakobson, op. cit.), con base en la pregunta que se formula Jakobson "¿qué es lo que hace que un mensaje verbal sea una obra de arte?"

Jakobson (ibid.: 348 ) señala que en tanto la poética se interesa por los problemas de la estructura verbal, es parte de la linguística. En la concepción general de los literatos es común que la poética y la linguiistica se distingan en una cuestiōn de valoración y, según Jakobson (ibid: 349 ) "esto se basa en la idea equivocada del contraste entre la estructura de la poesia y otros tipos de estructura verbal: éstos, se dice, se contraponen por su naturaleza 'casual', y carente de intención, al lenguaje poético, "no casual' e 'intencionado"'.

En los Cantares de Diztbalché, asi como en otros textos rituales indigenas coloniales y contemporáneos, encontramos un uso constante de expresiones que, debido a su caraicter ritual, tienden a ser marcadas discursivamente hablando. Las formas de discurso ritual generadas pueden ser analizadas bajo ciertos conceptos formales pertenecientes a la literatura occidental como la aliteración, repetición, sinonimia, anáfora, paralelismo, metáfora, difrasismo, ${ }^{6}$ metonimia, alegoria,

'Para Bricker y Edmonson (1985) estas frases que ellos denominan como 'Tenniags"; piensan que son propios del nábvatl y consideran que pudo haber sido un préstamo introducido en la literatura 
hiperbole y personificación, entre otras. En tanto que lo que me interesa es resaltar que la función poética del lenguaje se manifiesta con estructuras formales determinadas, en este trabajo me centraré sobre tres tipos de estructuras fónicas y sintácticas que se han considerado literarias, a saber, el paralelismo, la anáfora y la aliteración.

\section{¿Cómo se relacionan los Cantares de Dzitbalchẻ con las funciones del lenguaje?}

Partiendo de la idea de Jakobson (1984 |1956]: 358) de que el mensaje por el mensaje es la función poética del lenguaje y que ésta no es la única función del arte verbal, sino sólo su función dominante o determinante, podremos desarrollar a lo largo de este articulo los argumentos que sostienen la idea de que estos Cantares constituyen un texto poético. En éstos observamos un ritmo que está determinado en el verso, podemos ver cambio de énfasis en la persona gramatical expresada a veces en tercera persona (cantares 14 y 7 ), otras en segunda (15) y en otras ocasiones, en primera ( 4 y 7 ), lo que pone de relieve las diferentes funciones expresadas además de la poética, como son la referencial, la conativa y la emotiva. Aunado al cambio de persona gramatical que expresa distintas funciones, Jakobson (ibid.: 359) reconoce que para los géneros literarios la forma verbal expresada es la que indica cuál es la función que está siendo resaltada; de esta manera "la poesía épica, centrada en la tercera persona, implica con mucha fuerza la función referencial del lenguaje; la lirica, orientada a la primera persona, está intimamente vinculada con la función emotiva; la poesia de segunda persona está embebida de función conativa y es o bien suplicante, o bien exhortativa, según que la primera se subordine a la segunda o viceversa".

Si relacionamos los Cantares con formas gramaticales especificas (de primera, segunda y tercera persona, por ejemplo) y pensamos que estas formas expresan funciones particulares del lenguaje, también podemos relacionar estas funciones con los géneros literarios como ya señaló Jakobson (1984 [1956]). En tanto que los versos de los Cantores comparten una temática y tienen una estructura muy marcada respecto al habla conversacional - como el uso continuo de aliteraciones y paralelismos-, podemos considerar que si constituyen un género discursivo particular de tipo ritual, concerniente al ámbito del folklore más que al de la literatura. La aseveración anterior es pertinente si consideramos que los Cantares pertenecen al campo de la tradición oral, y como tales forman parte de la lengua como sistema.

Además de las personas gramaticales como marcadores de distintas funciones del lenguaje, la sintaxis es otro ámbito de la lengua en donde se manifiestan las figuras literarias, como señalaron Bricker y Edmonson (1985: 62):

yucateca. Consiste en un par semántico que, convencionalizado, tiene un tercer significado que puede no tener ninguna asociación con las palabras del difrasismo sueltas o fuera de este contexto. 
Several syntactic devices beside parallelism are common in Mayan literature. Normal, unmarked word order in Yocatec Maya is verb-object-subject (VOS) in transitive clauses and verb-subject [VS] in intransitive clauses. These word orders are frequently inverted in Mayan literature, through defting. Temporal adverbs normally follow verbs in Yucatec Maya, but temporal focus constructions, a characteristic of hieroglyphic texts, were abundant in Colonial Yucatecan literature.

De esta manera, a lo largo del trabajo podremos ver cómo se manifiestan las figuras literarias en todos los niveles de la lengua a través de distintas estrategias lingüisticas.

\section{Las manifestaciones lingüistico-formales en la estética de los Cantares}

En parrafos anteriores, discuti el tema del aspecto "literario" en los textos coloniales escritos en lenguas indigenas, y hable sobre el rigor metodológico con el que se debe abordar el estudio de éstos dentro del ámbito de la lingüistica. Por ejemplo, en los registros nahuas coloniales es muy recurrente el uso de difrasismos como in xochitt, in cuicatl 'flor y canto' o bien atl-tepetl 'agua-cerro', aunque son difrasismos recurrentes, no necesariamente se usan en contextos "literarios".

Hasta ahora, los documentos coloniales en lengua indigena han sido analizados bajo la óptica de los conceptos literarios de la cultura occidental. Por ello, es necesario, considerar distintos niveles de análisis, es decir, por un lado debemos considerar el lado puramente formal de los textos y por el otro, los aspectos semánticos-simbólicos. En el plano formal de los Cantores encontramos las marcas discursivas que responden a la estética de la lengua, es decir, formas que subrayan la función poética del lenguaje. En el nivel semántico-simbólico de cada Cantar el análisis se realiza bajo criterios de otro tipo, trabajo que Näjera (2007) realizó de manera exhaustiva.

Ahora bien, ya mencioné que en el tipo de discurso ritual de los Contares la función poética es la que predomina sobre las otras funciones del lenguaje y que su predominancia se refleja en distintos tipos de marcación. La manifestación formal de las figuras literarias en los Cantares se observa en los distintos niveles de la lengua, esto es, en el nivel fonológico, sintáctico y morfológico.

Comenzaré con la aliteración, "figura de dicción que consiste en la repetición de uno o más sonidos de formas en distintas palabras próximas. La aliteración es un fenómeno muy general que puede presentarse como distintas figuras literarias" (Beristáin, 1997 [1985]: 26, 27 y 28). Ésta puede manifestarse como cantidad y calidad vocalica. Según Jakobson (1984 |1956]), el uso de aliteraciones es algo que surge muy comúnmente en el habla cotidiana y tiene que ver con la paronomasia, esto es, por alguna razón el patrón silábico siempre influye en la selección de palabras y en el orden en el que las vamos a decir. Es por esto que el uso de aliteraciones no está ligado exclusivamente a los textos "literarios" sino 
que también pueden estar asociados a la poética de cualquier mensaje verbal entendiendo que "la función poética proyecta el principio de la equivalencia del eje de selección al eje de combinación" (Jakobson, 1984: 360).

A continuación presento ejemplos de distintas aliteraciones encontradas en el corpus. En primer lugar tenemos una aliteración especular del tipo/e-i-a/ /a-j-e/ y el contraste entre nasales y oclusivas: $/ \mathrm{m}-\mathrm{b}-\mathrm{k} / \mathrm{y} / \mathrm{k}^{\prime}-\mathrm{m}-\mathrm{n}-\mathrm{t}-\mathrm{k} /$

3. tumen binkah porque vamos

4. k'am nikte? al Recibimiento de la Flor

También aparece una aliteración con el uso de la palatal africada / $\bar{c} /$ en posición inicio de palabra en tres palabras de las dos siguientes lineas del Cantar 4:

7. cen cehlah [tienen en] pura risa

8. cehlameek y risa

Otros casos de aliteración se manifiestan en el Cantar 4 con el uso de las vocales altas $/ \mathrm{i} / \mathrm{y} / \mathrm{L} /$ alternando en las lineas 9 y 10 :

9. 7uyiic tut siit sus rostros, en tanto que saltan

10. 7upuksik'alil sus corazones

Por su parte, en el mismo Cantar 4 observamos una aliteración con el uso de la palatal fricativa $/ \mathrm{s} /$ en posición final de palabra. También interviene en la aliteración el uso del pluralizador -eé̉ en las lineas 43 y 44 :

43. kooš kooś konees Vamos, vamos, vámonos

44. palalees beey jôvenes; asi

En el mismo cantar vemos la aliteración provocada por el uso de la oclusiva velar sorda $/ \mathrm{k} /$ :

45. c'aik kiki? kimak daremos perfecto regocijo

Ahora veamos las aliteraciones del Cantar 7, en la linea 1 encontramos un ejemplo de éstas con el uso de la secuencia inicial casi idéntica en las dos palabras:

1. Tiških riskkitčpan La bellisima luna

Varios ejemplos de aliteraciones aparecen en las lineas 4 y 5 con la alternancia insistente de vocales $/ \mathrm{a} / \mathrm{y} / \mathrm{u} /$ por un lado, y el uso de la oclusiva velar sorda $/ \mathrm{k} / \mathrm{en}$ diversas posiciones, y de la africada palatal $/ \mathcal{C} /$ que se combina con su correlato glotalizado $/ \vec{c} /$ por el otro: 

4. tu cumuk kan kaan
en medio de los cielos
5. tuš kuç uuytal
donde queda en suspenso

Una aliteración similar a la anterior se observa en la línea 10 del Cantar 7 con el uso exclusivo de la vocal alta $/ \mathrm{w} / \mathrm{:}$

10. 7uc'uk'učul cumuk Ha llegado en medio

En la línea 19 del Cantar 7 encontramos la combinación de la secuencia final -il en tres palabras contiguas:

19. hel ?uyil kone?il le?il |nos| mirará

L.as líneas 47-53 del Cantar 7 expresan otro grupo de aliteraciones con el uso de la secuencia -eé en las que hay un intervalo regular de una palabra o dos que separan a aquellas que tienen esa secuencia:

47. nook'ees luusu?

48. kašil 7ahoolees

49. batenees hee?

50. kohikeeš way

51. yok'ool kabile?

52. 7issuhuyees

53. 7iscupalelees hed ?u viestras ropas, desatad

viestras cabelleras;

quedaos como

llegaiste aq̣uí

sobre el mundo.

virgenes,

mujeres mozas...

En el Cantar 14, encontramos varios grupos de aliteraciones. Para empezar cito el de la linea 1 que consiste en la combinación de la vocal baja/a/con consonantes oclusivas velares principalmente:

1. ti kak'ay čan sakpakal Alli cantas torcacita

De la misma manera aparece la combinación de las secuencias leyson/ con /iščn:

8. hee? beysan kolebil Asimismo ta Señora

9. yan yaalak hee? rišcan tiene sus aves: la pequeña

$Y$ en las lineas 12-14 las aliteraciones corresponden a dos tipos la secuencia

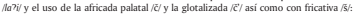

12. la>il Gan 7isc'unuun

13. la>il lariis 7uyaalak citc

14. i3kičpan šunan kolebil y cambién el colibri.

Son estas las aves

de la Belia Dueña y Señora 
En el Cantar 15, observamos una aliteración en las lineas 1-3-4: el sufijo -eex usado en varias palabras; la segunda aliteración se forma con el uso continuo de la sibilante / $/$ combinada con su correlato glotalizado. Una vez más, vemos que la africada palatal y la fricativa / $/$ / alternan en contexto final de palabra de distintas palabras:

1. c'a?ees 7ahac'uutc nok'ees

3. seeč 7ucow coeel 7apol

4. c'à? ?ulemkeeć kiič kel mil
Poneos vuestras bellas ropas; peinad la maraña de vuestra cabellera poneos la más bella

Y en las lineas 16-19 observamos el mismo patrón de aliteración con las mismas consonantes y secuencias que en los ejemplos anteriores:

16. Pinkaat kaylabeec haac

17. sempeec kiçpameec tumen ku

18. yan kaciikpakeeć ti ?isbuuc

19. 7ek tumen kaw c'iboolteectak quiero que seias vista en verdad muy bella porque habréis de pareceros a la humeante estrella; porque os deseen hasta

En las líneas 9-10 del Cantar 15 se combinan en posición final las consonantes labiales $/ \mathrm{b} / \mathrm{y} / \mathrm{p} /$, con la velar $/ \mathrm{k} /$ y con la vocal baja $/ \mathrm{a} /$ :
9. 7a?iskictpan kaal c'aw 7ubaak'al
de vuestra bella garganta;
10. hop men hop tu nak 7ak'ab poned lo que enroscalis y reluce en la parte rolliza de vuestros brazos.

Las aliteraciones presentes en los Cantares en lineas contiguas o bien, en el interior de un mismo párrafo, son un recurso común en los diferentes cantares. Es interesante ver que el uso de este recurso no se ve sólo en relación con los versos de cada cantar, sino que el uso de los grupos de secuencias vocálicas con la misma calidad y con consonante en coda: iiC, o eeC a final y entre los párrafos son recurrentes en los cantares 4 y 15 , es decir, intertextualmente. La idea de que estos textos fueron escritos justamente para cantarse, sugiere y justifica aún más la intención de realizar estas aliteraciones.

Otra figura encontrada es la anáfora? que en palabras de Beristáin (1997 |1985|: 40-41) es una "figura de construcción porque afecta la forma de las frases. Consiste en la repetición intermitente de una idea, ya sea con las mismas o con otras palabras. Los pronombres suelen cumplir esta función (gramatical). pero también otra clase de palabras como los adverbios".

\footnotetext{
${ }^{3}$ La andifora en linguística tiene otro sentido y no necesarianente implica la repetición de la misma palabra, sibo una correferencia con un referente dado, en este texto debe considerarse la de: finición que està escrita arriba.
} 
En el Cantar 14 también encontramos tres formas anaföricas dentro de un mismo parrafo:

7. Tuyaalak'oob yum k'uh

9. yan yaalak' hee? 7išcan

13. La?l la?iis ?uyaalak' ciec
La aves del Señor Dios.

tiene sus aves: la pequefia

Son estas las aves

De manera más clara en el Cantar 15 aparece la repetición del verbo c'a? traducido como 'poner' en varias partes del pasaje a manera de anáfora.

1. c'a 'ees ?ahac'uute nok'ees

4. c'a? rulemkeeć kicic kel mil

5. 7anok' c'a? hac'uc șanab

7. tuup tu tupil 7asikin e'a?

8. malob 7ooc' c'a> 7uk'eestoob

9. 7aiskiićpan kaal c'a?
Poneos vuestras bellas ropas:

poneos la más bella

de vuestras ropas; poneos vuestro

bello calzado:

pendientes en los pendientes veestras

orejas, poneos

buena toca: poned los galardones

de vuestra bella garganta; poaed lo que

Aunque no necesariamente aparecen en posición inicial, en los pasajes anteriores podemos observar el uso repetido de dos palabras en el interior de un mismo parrafo. El uso estético justamente radica en la repetición y como tal, forman parte del sistema de la lengua.

Ahora bien, continuemos con el paralelismo que, según Beristäin (1997 [1985]: 389-390). "se trata de la relación espacialmente equidistante o simétrica que guardan entre si las estructuras repetitivas de los significantes y/o de los significados, y en virtud de la cual se revelan las equivalencias fónicas (como en la similicadencia), morfológicas (como en la anáfora), sintáctico-semánticas o semánticas como en la sinonimia, y se revela tambièn la red de relaciones que determina la estructura del texto....

Para Jakobson (1984 [1956]: 378) existen dos tipos de paralelismo: de oposición marcada, o de oposición cromática o transicional, y sólo el primero se relaciona con la estructura del verso:

en el ritmo, la repetición de una cierta secuencia de silabas determinada: en el metro, Ja repetición de una cierta secuencia del titmo; en la aliteración, en la asonancia y en la rima. Ahora bien, la fuerza de esta repetición consiste en engendrar una repeticion o paralelismo que le corfesponda en palabras o ideas, y, hablando en general y más como tendencia que como resultado invariable, un paralelismo más marcado, ya en su estructura, ya en su elaboración o en su tono, engendrará un paralelismo más marcado en las palabras y en el sentido. Al tipo de paralelismo marcado o saliente corresponden la metáfora, el simil, la parábola, etc. En donde el efecto se busca en la semejanza de las cosas, y en la antítesis, el contraste, etc., en donde se busca la desigualdad. 
En el Cantar 15 encontramos una expresión que formalmente expresa un paralelismo: comparte el inicio de una expresión, la forma hop que en el Diccionario Maya Condemex: 231 aparece como avivar el fuego, hacerlo brillar, que levante la Ilama". Aunque la traducción no sugiere nada relacionado con anáfora, esta forma y la variación de la forma léxica que le sigue a esta raiz hace pensar en la posible repetición de algún evento:

10. hop men hop tu nak 7ak'ab reluce en la parte rolliza de vuestros brazos.

En esta frase en particular, vemos que la traducción no sugiere nada respecto al uso intencional de la palabra hop para expresar una forma estética, pero una vez que observamos con cuidado el texto original en maya podemos ver cosas que en la traducción no se resaltan, justo como el caso de la repetición de esta forma como un recurso estético expresado como paralelismo.

De la misma manera en el verso 3 del Cantar 15 aparece un paralelismo en el interior de un mismo verso y, si no atendemos a la traducción sino a la forma coc 'cabello':

3. 3eec ?ucow cocel 7apol peinad la maraña de vuestra cabellera

En el Cantar 4 encontramos otro paralelismo con la forma celhlah que notablemente expresa también una aliteración con la repetición de la secuencia de sonidos /ce/. La segmentación de la forma que aparece en el verso 8 no es la adecuada pues parece que la palabra es čhlah, que viene de $\bar{c} e$ ? $h$, 'risa' $\langle$ Cordemex: 85 ] y es justo la que se repite en construcción paralela:

7. cen cehlah [tienen en] pura risa

8. ceblameek y risa

Otros textos paralelos del Cantar 4 los observamos en las siguientes lineas:

25. tu taan 7issuhuy ante la Virgen

26. 7iškiicpan suhuy la Bella Virgen

39. kuštalil way yok' a la Vida aquí sobre

40. peetene? way yok' la Región, aquí sobre

En el Cantar 14 tenemos otra estructura paralela:

21. cen k'ay cen bakaal isolo cantos, solo juegos

En el Cantar 7 encontramos estos dos grupos de paralelismos, pero como podemos ver, este tipo de frases paralelas juegan también con el recurso de la 
anáfora, ya que es la repetición de una palabra en posición inicio de frase la que dispara los paralelismos:

25. beysan 7izkok bos

26. beysan tumben his

28. tumben kuc

tumben luc

29. bolom yaaš took'

30. tumben peec'ilil

31. tumben soot beyšan

32. 7ukan 7is?ulum

33. tumben sanab tulakal tumben lasil sam ?ukašil así como la concha de la tortuga terrestre

asimismo el nuevo polvo de calcita

el nuevo hilo para hilar; La nueva jicara

$y$ el grande $y$ fino pedernal;

la nueva pesa:

La nueva tarea de hilado;

el presente del pavo.

nuevo calzado, todo nuevo,

inclusive las bandas que atan

Las siguientes lineas del Cantar 4 , expresan un paralelismo de forma y contenido, ya que las formas 7iskiiçpan e ?issuhury comparten algunos rasgos semánticos, de tal suerte que tienen una traducción aproximada de 'mujer bella' y forman construcciones paralelas en estos pasajes:

32. beysan riskiçpan

33. 7isk 'amleooc

34. 7iškiiçpan 7išah

35. 7oot yetel 7iskiliçan

36. kolel Pissuhuy $7 i s ̧$
Asimismo fantel la Bella

$x$ Kanleox

y |ante| La Bella X z

oot $y$ la Bella

Señora Virgen $x$

Cuando observamos los Cantares desde la perspectiva lingüistico-poética vemos que el valor estético de la lengua se hace explicito a través de estructuras discursivamente marcadas que expresan figuras literarias. En la aliteración, por ejemplo, la marcación se queda en el nivel fónico. En el paralelismo y la anáfora se expresan una combinación de elementos sintáctico-semánticos que son dificiles de analizar como unidades separadas pues sólo cobran sentido cuando se combinan en el nivel de frase o de oración. El análisis de estas dos figuras es mucho más rico aùn cuando las observamos a través de los distintos niveles lingüisticos, esto es, desde el fonológico hasta el sintáctico. Es por eso que Jakobson (1984 [1956]) menciona la importancia de entender la poética como una parte de la lingüistica en la que las distintas funciones del lenguaje entran en juego y enfatizan, en el caso de un texto poético, el discurso mismo. 


\section{Consideraciones finales}

Al realizar este trabajo me encontré con que una de las principales dificultades para abordar los Cantares es que la traducción presentada por Barrera (1980) no es adecuada para realizar un análisis lingüistico-poético, pues como vimos, en muchos casos ésta no corresponde con lo que se está expresando en maya. Esto tiene como consecuencia que el lector ponga toda su atención en el texto traducido y no se centre en las formas expresadas en el maya yucateco y que finalmente, no haga de su objeto de estudio la lengua como tal sino que se enfoque en la interpretación del autor. Al mismo tiempo, esto provoca que el lector pase por alto las estructuras formalmente marcadas en la lengua maya, que expresan una estética particular y que puede ser objeto de estudio per se.

En la actualidad existen esfuerzos importantes por parte de los linguiistas por establecer una metodología de traducción de lenguas indigenas en la que se trate de respetar en primer lugar los conceptos y expresiones estéticas en la lengua original, en las que el lector enfatice el ritmo y los recursos estéticos originales de la lengua en cuestión. Valiéndose de notas a pie y comentarios al margen, el traductor puede agregar información que le parezca pertinente en un esfuerzo por realizar traducción cultural además de lingüistica.

Para proponer una nueva traducción del texto se requiere de un análisis lingüistico sistemático, y del análisis del maya yucateco desde una perspectiva sincrónica enfocada en el siglo xom. Este nuevo análisis requiere también del cruce de información con otro tipo de datos, los mitos y tradiciones coloniales, y de la lengua maya colonial, asi como algunas posibles representaciones de los primeros que soporten de manera sólida los argumentos para proponer una traducción y análisis que refleje de manera más cercana la cultura y lengua maya.

\section{BIBLIOGRAFLA}

Barrera Visquez, Alfredo

1980 E. libro de las Cantares de Deitboíché, traducción, intraducción y notas de... Mérida: Ayuntamiento de Mérida Ediciones.

Beristáin, Eena

1997 [1985] Diccibuario de Retúrica y Poética. México: Porrúa.

Clements, George N., y Samuel Jay Kayser

1983 CV Phowology. A Generathe Theory of the Syllable. Cambridge, Massachusetts: The MrT Press.

Dicrionerio Maya Cordentex

1980 Edición de Alfredo Barrera Vásquez. Mérida: Cordemex. 
Edmonson, Munro 5 .

1982 "The Songs of Dzitbalche: A Literary Commentary", Tiafocan. Revista de foentes para el conocimiento de las culturas indigenas de Mésica, IX: 173-20B, México: UNAM, Instituto de Investigaciones Históricas/Instituto de Investigaciones Filologicas.

y Victoria R. Bricker

1985 "Yucatecan Mayan Literature", Handbook of Middle American Indians Supplement, Munro Edmonson (ed.), 3: 44-63. Austin: University of Texas Press.

Jakobson, Roman

1984 [1956] "Lingtística y poética", Ensoyos de lingüistica general. Batcelona: Ariel.

Lacadena, Alfonso

(s. f.) "Apuntes para un estudio sobre literatura maya antigua", para ser publicado en Texto y contexto: Perspectives intracuifurales en el andisis de la literatura maya yucateca, A. Cunsenheimer, Ts. Okoshi y J.F. Chuchiak (eds.). Bonts: BAS (Estudios Americanistas de la Universidad de Bonn).

Lázaro, Femando

1986 Estudios de poetica (lie abra ent si). Madrid: Taurus.

Mukarovsky, Jan

1976 On Poetic Language. New Haven-Londres: The Peter and the Ridder Press.

Najera, Marta llia

2007 Las cantares de Dzitbaiché en fo trodición religiosa mesoamericana. Méxica: UNAM, Instituto de Investigaciones Filológicas.

Vail, Gabriela, y Andrea Stone (eds.)

2002 "Representation of Women in Postclassic and Colonial Maya literature and Art", Ancient Maya Women, Traci Ardren |ed.|. Lanham, Maryland: Altamira Press.

Vidal Beneyto, José (ed.)

1981 Posibilidades y timites del andilsis estructural. Unte investigacien cancreta en torno al lenguaje y poesía. Madrid; Editora Nacional. 

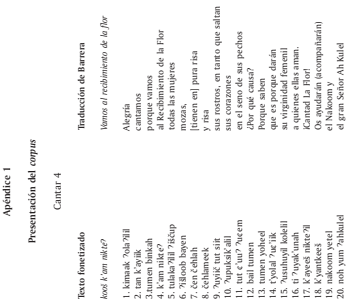

告

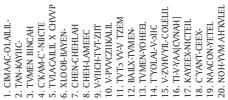




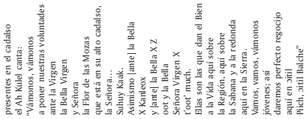

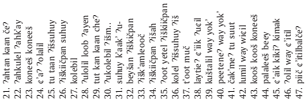

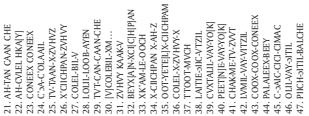




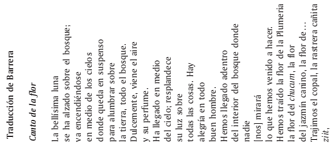

采

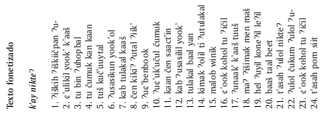

를

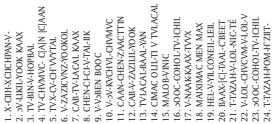



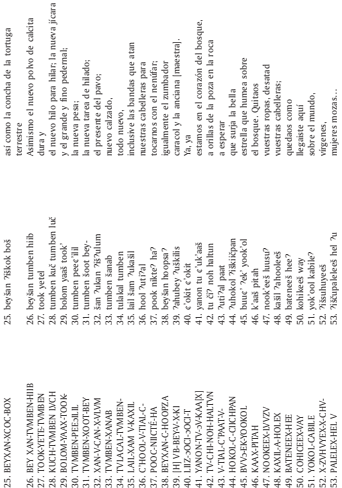


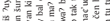

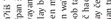

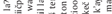

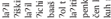
10 

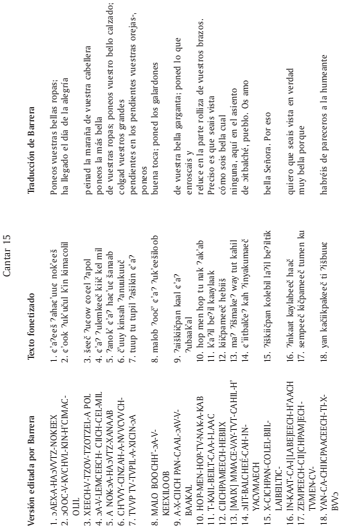


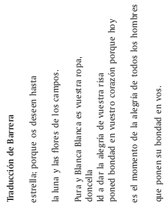

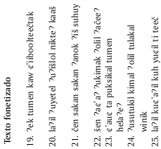

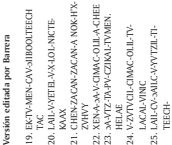

